\title{
A FIELD THEORETIC APPROACH TO THE ELECTRIC INTERFACIAL LAYER. MIXTURE OF TRIVALENT ROD-LIKE AND MONOVALENT POINT-LIKE IONS BETWEEN CHARGED WALLS.
}

\author{
KLEMEN BOHINC \\ Faculty of Health Sciences, University of Ljubljana, \\ Zdravstvena 5, 1000 Ljubljana, Slovenia \\ klemen.bohinc@zf.uni-lj.si
}

LEO LUE

Department of Chemical and Process Engineering, University of Strathclyde James Weir Building, 75 Montrose Street, Glasgow G1 1XJ, United Kingdom leo.lue@strath.ac.uk

Received (Day Month Year)

Revised (Day Month Year)

\begin{abstract}
In this work, we study the interaction between charged particles immersed in a solution of oppositely charged, rod-like counterions and point-like co-ions. The system is modeled by a field theoretic approach, which was extended to treat mixtures of rod- and point-like ions. The theory is applicable from the weak to the intermediate through to the strong coupling regimes. In the weak coupling limit, the force between the charged surfaces are only repulsive. In the intermediate coupling regime, the rod-like ions can induce attractive force between the charged surfaces. In the strong coupling limit, the inter-ionic charge correlations dominate the attractive forces at short separations between the charged surfaces.
\end{abstract}

Keywords: Field theory; Interfacial layer; Electrostatic interactions.

\section{Introduction}

Electrostatic interactions are ubiquitous in biological and colloidal systems; consequently, it is of fundamental importance to understand the interactions between charged macromolecules in electrolyte solutions ${ }^{1}$. These interactions are especially important in stabilizing colloidal systems, including sols and clays ${ }^{2}$. Colloids in aqueous solutions can acquire charges via ionization of surface acid or base groups, thereby becoming macroions with charged surfaces. Ion adsorption or desorption onto a macroion surface creates an electric interfacial layer. In clays, charge arises by isomorphous substitution of one atom by another.

In the absence of counterions, like-charged objects repel each other. The presence of counterions, however, can lead to an intuitively surprising effective attrac- 
tion between like-charged macroions in solution. For example, this attraction can be mediated via the correlated motion of mobile multivalent ions in the solution 3 . Hexagonal arrays of double stranded DNA are formed by polyamines and other ions with a valency higher than two; even some divalent ions like manganese and cadmium can form DNA arrays ${ }^{4}$. Divalent diamin ions can induce aggregation of rod-like M13 viruses ${ }^{5}$, whereas divalent barium ions can mediate network formation in actin solutions ${ }^{6}$. Divalent ions can also induce adsorption of DNA onto zwitterionic lipid layers ${ }^{7}$. Multivalent ions which possess a spatial charge distribution usually show a strong tendency to induce aggregation of like-charged macroions. Example systems include positively charged colloids that condense DNA ${ }^{1}$ and DNA that induces attraction between cationic lipid membranes ${ }^{8}$.

The presence of an attractive force between two equally charged surfaces immersed in a solution composed of divalent ions in the limit of high surface charge density was first calculated by Monte-Carlo (MC) simulations of Guldbrand et al. ${ }^{12}$. Subsequently, more detailed MC simulations ${ }^{13,14,15}$ showed that attractive interactions between equally charged surfaces may arise for high surface charge density, low temperature, low relative permittivity and multivalent counterions. The classic Poisson-Boltzmann (PB) theory predicts only repulsive interactions between equally charged surfaces ${ }^{9}$. The $\mathrm{PB}$ theory is a mean field theory where ions are treated as point-charges and the solvent is considered a uniform, continuous dielectric medium. Charge-charge correlations must be considered in order to describe attractive interactions between like charged surfaces in electrolyte solutions ${ }^{10,11}$.

In real solutions, ions usually have an internal structure, with individually separated charges and with possibly additional rotational degrees of freedom. Charge correlations as a result of bonding between charges within an ion (e.g., the intraionic correlations induced by the fixed distance between charges in a rod-like ion) can be sufficient to change repulsive into attractive interaction between like-charged surfaces ${ }^{18,19,22}$. In these weak coupling studies, the rod-like ions carry a single elementary charge on each end. The minimum of the free energy occurs for the rod-like counterions oriented perpendicularly to the like-charged surfaces. The rod-like ions bridge left and right oppositely charged surfaces. Monte Carlo simulations confirm these theoretical predictions ${ }^{19,22,23}$. Also rod-like dipoles and spherical quadrupoles between charged surfaces have also been studied ${ }^{20,21}$.

The description of systems of rod-like ions has been extended to the intermediate and strong coupling regimes, where the interionic correlations alone can lead to an attraction between like-charged surfaces ${ }^{24,25,26}$. In systems with highly charged macroions and mobile multivalent ions, correlations between the charges need to be introduced $27,28,29$. In the description of strongly coupled systems, the field theoretic approach has shown to provide good predictions, in comparison to simulation results.

In this work, we present a general field theoretic method to describe ions with a spatially distributed charge confined between large, highly charged macroions in a planar geometry. First, we consider a mixture of trivalent rod-like 
counterions and monovalent point-like co-ions. Second we reduce the system to only trivalent rod-like ions, and third we examine point-like trivalent ions. The influence of monovalent point-like counterions on the trivalent rod-like profiles is considered. Further, we examine how the charge distribution on rod-like counterions influences the interaction between two similarly charged surfaces and how they differ from point-like trivalent counterions.

\section{Theory}

We analyze systems of rod-like ions and point ions that are confined between two charged plates separated by a distance $D$. Each rod has a total length $l$ and consists of 3 rigidly bonded and equally spaced point charges of magnitude $q$. The charge density $Q_{R}(\mathbf{r}, \hat{\mathbf{n}})$ of one of these rods, with one end located at the origin and the final point charge located at a position $l \hat{\mathbf{n}}$, is given by

$$
Q_{R}(\mathbf{r}, \hat{\mathbf{n}})=q \sum_{k=0}^{2} \delta^{d}\left(\mathbf{r}-k l^{\prime} \hat{\mathbf{n}}\right),
$$

where $l^{\prime}=l / 2$ is the distance between neighboring point charges. The charge density of a monovalent point-like ion is given by $Q_{P}(\mathbf{r}, \hat{\mathbf{n}})=-q \delta^{3}(\mathbf{r})$. The charge of the rod-like and point ions is neutralized by a fixed surface charge density $\Sigma$ on the confining plates. The total charge density $\mathcal{Q}(\mathbf{r})$ of the system composed of mobile ions and a fixed charge distribution is

$$
\mathcal{Q}(\mathbf{r})=\sum_{j} Q_{R}\left(\mathbf{R}_{j}, \hat{\mathbf{n}}_{j}\right)-\sum_{k} q \delta^{3}\left(\mathbf{r}_{k}\right)+\Sigma \delta(z)+\Sigma \delta(z-D)
$$

where $\mathbf{r}_{k}$ is the position of point charge $k, \mathbf{R}_{j}$ is the position of rod $j$, and $\hat{\mathbf{n}}_{j}$ is its orientation.

The electrostatic energy $E$ of the system is

$$
E_{e l}=\frac{1}{2} \int d \mathbf{r} d \mathbf{r}^{\prime} \mathcal{Q}(\mathbf{r}) G\left(\mathbf{r}, \mathbf{r}^{\prime}\right) \mathcal{Q}(\mathbf{r})
$$

where $G\left(\mathbf{r}, \mathbf{r}^{\prime}\right)=\epsilon^{-1}\left|\mathbf{r}-\mathbf{r}^{\prime}\right|^{-1}$ is the Green's function of the Poisson equation. Physically, the Green's function represents the electrostatic potential at position $\mathbf{r}$ due to a unit point charge located at position $\mathbf{r}^{\prime}$.

A key length scale that characterizes theses systems is the Bjerrum length. It is defined as $l_{B}=q^{2} /\left(\epsilon k_{B} T\right)$, where $\epsilon$ is the dielectric constant of the solvent (water), and $k_{B} T$ is the thermal energy. We use Gaussian units for which $4 \pi \epsilon_{0}=1$, where $\epsilon_{0}$ is the permittivity of free space. Due to the high dielectric constant of water $(\epsilon=80)$, ions in aqueous solutions often dissociate and become mobile. This high dielectric constant greatly reduces cation-anion attraction and favors the entropic gain that comes with the dissociation process. Besides the distance between the walls $D$ and the length of rod-like ions $l$, an additional length scale is the GouyChapman length $\mu=q /\left(2 \pi l_{B} \Sigma\right)$. It measures the distance at which the 
thermal energy equals the counterion-wall interaction energy and gives a measure of the thickness of the counterion layer at a charged wall. The ratio between Bjerrum length and Gouy-Chapman length is the coupling parameter $\Xi=l_{B} / \mu$, which characterizes the strength of the electrostatic interactions.

Field theory based approaches work well for long-wavelength fluctuations. On the other hand, particle based approaches, such as virial expansions, work well for short-wavelength correlations. In the splitting approximation ${ }^{24,25}$, we divide the fluctuations of the system into short-wavelength and long-wavelength contributions and treat each of these within different approximation schemes. This leads to a theory which works well for both the weak coupling and strong coupling limits, as well as for intermediate coupling strengths. Technically, we divide the Green's function of the electrostatic interactions into short-wavelength $G_{s}$ and long-wavelength $G_{l}$ contributions as ${ }^{24,25,26}$ :

$$
G\left(\mathbf{r}, \mathbf{r}^{\prime}\right)=G_{s}\left(\mathbf{r}, \mathbf{r}^{\prime}\right)+G_{l}\left(\mathbf{r}, \mathbf{r}^{\prime}\right)
$$

where $G_{l}=\mathcal{P} G$, and $G_{s}=(1-\mathcal{P}) G$, and $\mathcal{P}$ is an operator that projects out the long-wavelength components of a function. The form of this operator is fairly arbitrary, and in this work, we use $\mathcal{P}=\left[1-\sigma^{2} \nabla^{2}+\sigma^{4} \nabla^{4}\right]^{-1}$, where the splitting parameter $\sigma$ is a length scale which separates short-wavelength and long-wavelength regimes. Physically, $\sigma$ is the radius of the correlation hole around each of the charges and is determined by making the free energy stationary.

The grand partition of the system can be written as an integral over all possible positions and orientations of the particles in the system. Another way to represent the grand partition function is to transform it to a functional integral over interaction fields. In this approach, the focus is changed from particles to the interaction potential generated by the particles, which is performed mathematically using the Hubbard-Stratonovitch transformation. This transformation is done separately for the short-wavelength and long-wavelength components of Green's function, which leads to functional integrals over associated short-wavelength and long-wavelength fields. The averages over these fields are performed using different approximations. The short-wavelength field $\psi_{s}$ is strongly fluctuating and averages over this field are approximated by a truncated cumulant expansion. The results are equivalent to a virial series in which the particles interact with an effective one and two body potential. The long-wavelength field $\psi_{l}$ is weakly fluctuating. A single configuration of the long-wavelength field dominates the contribution to the partition function. Configurations deviating substantially from main configuration do not make a significant contribution. Averages over this field are performed using the mean field approximation. 
The approximate Helmholtz free energy $F$ of the system can be written as

$$
\begin{aligned}
& \beta F[\rho, \Sigma] \approx \int d \mathbf{R} d \hat{\mathbf{n}} \rho_{R}(\mathbf{R}, \hat{\mathbf{n}})\left[\ln \rho_{R}(\mathbf{R}, \hat{\mathbf{n}}) \Lambda_{R}^{3}-1\right]+\int d \mathbf{r} \rho_{P}(\mathbf{r})\left[\ln \rho_{P}(\mathbf{r}) \Lambda_{P}^{3}-1\right] \\
&-\frac{1}{8 \pi} \int d \mathbf{r} \epsilon \nabla \phi(\mathbf{r}) \cdot \nabla i \bar{\psi}_{l}(\mathbf{r})+\int d \mathbf{r}[\Sigma(\mathbf{r}) \\
&+\int d \mathbf{R} d \hat{\mathbf{n}} \rho_{R}(\mathbf{R}, \hat{\mathbf{n}}) Q_{R}(\mathbf{r}-\mathbf{R}, \hat{\mathbf{n}}) \\
&\left.+\int d \mathbf{R} \rho_{P}(\mathbf{R}) Q_{P}(\mathbf{r}-\mathbf{R})\right] i \bar{\psi}_{l}(\mathbf{r}) \\
&+\int d \mathbf{R} d \hat{\mathbf{n}} \rho_{R}(\mathbf{R}, \hat{\mathbf{n}}) \beta u_{R}(\mathbf{R}, \hat{\mathbf{n}})+\int d \mathbf{r} \rho_{P}(\mathbf{r}) \beta u_{P}(\mathbf{r})+\beta E_{s}^{\mathrm{se}}
\end{aligned}
$$

where $\Lambda_{R}$ is the de Broglie wavelength of the particles, $\Lambda_{P}$ is the de Broglie wavelength of the point ions, and $E_{s}^{\text {se }}$ is the short-wavelength contribution to the electrostatic interaction energy. The one-body interaction potential of the rods is explicitly given by

$u_{R}(\mathbf{R}, \hat{\mathbf{n}})=q \sum_{k=0}^{2} \int d \mathbf{r}^{\prime} G_{s}\left(\mathbf{R}+k l^{\prime} \hat{\mathbf{n}}, \mathbf{r}^{\prime}\right) \Sigma\left(\mathbf{r}^{\prime}\right)-\frac{q^{2}}{2} \sum_{j, k=0}^{2} G_{l}\left(\mathbf{R}+j l^{\prime} \hat{\mathbf{n}}, \mathbf{R}+k l^{\prime} \hat{\mathbf{n}}\right)+v_{R}(\mathbf{R}, \hat{\mathbf{n}})(5)$

where $v_{R}(\mathbf{R}, \hat{\mathbf{n}})$ is an applied non-electrostatic potential. The one-body interaction potential of the point ions is given by

$$
u_{P}(\mathbf{r})=-q \int d \mathbf{r}^{\prime} G_{s}\left(\mathbf{r}, \mathbf{r}^{\prime}\right) \Sigma\left(\mathbf{r}^{\prime}\right)-\frac{q^{2}}{2} G_{l}(\mathbf{r}, \mathbf{r})+v_{P}(\mathbf{r})
$$

where $v_{P}(\mathbf{r})$ is an applied non-electrostatic potential on the point charge.

Minimization of the free energy of the system with respect to the mean value of the long-wavelength field $i \bar{\psi}_{l}$ leads to the Poisson equation

$$
-\frac{\epsilon}{4 \pi} \nabla^{2} \phi(\mathbf{r})=q_{R}(\mathbf{r})+q_{P}(\mathbf{r}),
$$

with the mean electric potential $\phi(\mathbf{r})=\beta^{-1} \mathcal{P}^{-1} i \bar{\psi}_{l}(\mathbf{r})$ and the charge density of the rods and point charges given by

$$
\begin{aligned}
& q_{R}(\mathbf{r})=q \sum_{k=0}^{2} \int d \hat{\mathbf{n}} \rho_{R}\left(\mathbf{r}-k l^{\prime} \hat{\mathbf{n}}, \hat{\mathbf{n}}\right) \\
& q_{P}(\mathbf{r})=-q \rho_{P}(\mathbf{r})
\end{aligned}
$$

with

$$
\begin{aligned}
\rho_{R}(\mathbf{R}, \hat{\mathbf{n}}) & =\Lambda_{R}^{-3} e^{\gamma_{R}(\mathbf{R}, \hat{\mathbf{n}})-\beta u_{R}(\mathbf{R}, \hat{\mathbf{n}})-q \sum_{k} \bar{\psi}_{l}\left(\mathbf{R}+k l^{\prime} \hat{\mathbf{n}}\right)} \\
\rho_{P}(\mathbf{r}) & =\Lambda_{P}^{-3} e^{\gamma_{P}(\mathbf{R}, \hat{\mathbf{n}})-\beta u_{P}(\mathbf{R}, \hat{\mathbf{n}})+q \bar{\psi}_{l}(\mathbf{r})}
\end{aligned}
$$


Fig. 1. A schematic illustration of two like charged planar macroions embedded in a solution of trivalent rod-like and monovalent point-like ions.

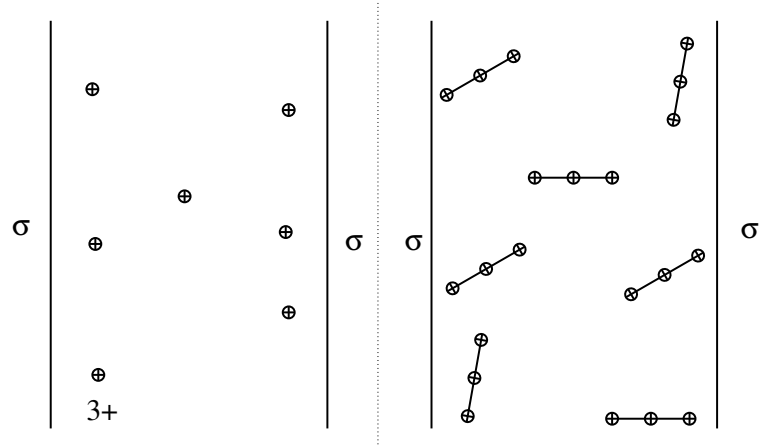

Fig. 2. A schematic illustration of two like charged planar macroions embedded in a solution of (a) spherical trivalent ions and (b) trivalent rod-like ions.

\subsection{Results and discussion}

We now examine a system of trivalent rod-like counterions and monovalent point co-ions between two like-charged surfaces (see Fig. 1). Later we reduce the system to trivalent rod-like ions (see Fig. 2b). We also study systems of trivalent point charges (see Fig. 2a) confined between charged plates. The length of rod is $1 \mathrm{~nm}$, with two elementary charges located at each end of the rod and one elementary charge located at its center. The system is immersed in water, with the Bjerrum length of $0.7 \mathrm{~nm}$. The following analysis is based on numerical solutions of integro-differential equations presented in the previous section.

Fig. 3. Field theoretic results. Density distribution of trivalent rod-like counterions of length $l=1 \mathrm{~nm}$ and monovalent point-like counterions confined between two plates. Left: $\Sigma=0.02 \mathrm{C} / \mathrm{m}^{2}$, right: $\Sigma=0.2 \mathrm{C} / \mathrm{m}^{2}$. Full lines: bulk counterion concentration $10^{-2} \mathrm{M}$, dashed lines: bulk counterion concentration $10^{-4} \mathrm{M}$. The separation between charged surfaces is $D=2 \mathbf{n m}$.

Figure 3 shows the density distribution of trivalent rod-like counterions and monovalent point-like co-ions for two different surface charge densities and two different counterion bulk concentrations. We observe that the increasing surface charge $\Sigma$ of the plates significantly increases 
the ion density near the plates. The reason is the strong electrostatic attraction between negatively charged surfaces and positive trivalent rod-like counterions. The steric restriction prevent that rod-like counterions could not penetrate through the charged walls. The kink in the ion density profiles for the rods at a distance $l$ from the surface results also from the rotational restriction. Point-like monovalent counterions are attracted by the charged surface to less extent. In this range of concentrations, the influence of monovalent point-like counterions is small.

Fig. 4. Field theoretic results. Density distribution of trivalent rod-like counterions of length $l=1 \mathrm{~nm}$ (full lines) and point counterions (dashed lines) confined between two plates with charge density $\Sigma=0.2 \mathrm{C} / \mathrm{m}^{2}(\mathrm{a}, \mathrm{c})$ and $\Sigma=0.02 \mathrm{C} / \mathrm{m}^{2}(\mathrm{~b}, \mathrm{~d})$. The separation between charged surfaces is $D=2 \mathrm{~nm}$.

Figure 4 shows the density distribution of trivalent rod-like and point counterions for two different surface charge densities. The solid lines corresponds to rod-like counterions whereas the dashed lines corresponds to point-like counterions. In all cases, the MC results are in a good agreement with the theory. It can be seen that increasing the surface charge $\Sigma$ of the plates significantly increases the ion density near the plates. The ion density at the charged surface is lower for the rod counterions as compared to the point counterions, at the same conditions. This is due to the rotational restriction for rod counterions close to the surface, which leads to a relative depletion of the counterions. The kink in the ion density profiles for the rods at a distance $l$ from the surface results also from the rotational restriction. In the case of trivalent point-like ions and small surface charge densities, the concentration profiles obtained by the PB theory agrees quite with the field theoretic predictions. The discrepancy becomes important for very large surface charge densities.

Figure 5 shows the equilibrium distance between two like charged surfaces as a function of surface charge density $\Sigma$. The curves represent the conditions at which the pressure between the plates is zero. The regions between the curves separate the attractive from the repulsive interactions between like charged surfaces. The repulsive attraction is obtained for smaller surface charge densities and smaller equilibrium separations between the charged surfaces, as well as for larger surface charge densities and larger equilibrium separations between the charged surfaces.

Fig. 5. Equilibrium plate separation $D_{\text {equil }}$ as a function of the surface charge density for trivalent rod-like ions (full line) and for trivalent point-like ions (dashed line). The regions of attractive and repulsive interaction between like charged surfaces is shown. 


\section{Conclusions}

In conclusion, we examined the influence of charge distribution within particular ion on the interaction between like charged surfaces. The study was made for a mixture of trivalent rod-like ions and monovalent point-like counterions. We have used a previously developed field theory approximation which is known to provide accurate predictions for multivalent ions. The theoretical results are in good agreement with Monte Carlo simulation results.

Generally, for sufficiently long rod-like counterions, there are two distinct regions of attractions between the plates. One occurs at plate separations that are about the length of the rods (bridging of the counterions across both plates). In addition to bridging, an attractive region occurs at lower surface separations (correlations between the counterions). With decreasing length of the rod-like counterions, the attractive region associated with "bridging" prolonged to shorter surface separations.

The results in this work may be verified experimentally using surface force apparatus and atomic force microscopy to measure the force between like charged surfaces. The theoretical results can also give new insights for the study of interactions between DNA molecules induced by trivalent rod-like ions like spermidine (DNA condensation) ${ }^{4}$.

\section{References}

1. W. M. Gelbart, R. Bruinsma, P. A. Pincus and V. A. Parsegian, Physics today 53 (2000) 38.

2. R. J. Hunter, Foundations of Colloid Science (Oxford University Press, Oxford, 1987).

3. D. F. Evans and H. Wennerström, The colloidal domain, where physics, chemistry, biology and technology meet, 2nd ed. (VCH Publishers, New York, 1994).

4. V. B. Teif and K. Bohinc, Prog. Biophys. Mol. Biol. 105 (2011) 208.

5. J. C. Butler, T. Angelini, J. X. Tang and G. C. L. Wong, Phys. Rev. Lett. 91 (2003) 028301.

6. T. E. Angelini, H. Liang, W. Wriggers and G. C. L. Wong, Proc. Natl. Acad. Sci. U S A 100 (2003) 8634

7. K. Bohinc, G. Brezesinski and S. May, Phys. Chem. Chem. Phys. 14 (2012) 10613.

8. J. O. Rädler, I. Koltover, T. Salditt and C. R. Safinya, Science 275 (1997) 810.

9. S. A. Safran, Statistical thermodynamics of surfaces, interfaces, and membranes (Westview Press, Colorado, 2003).

10. S. Carnie and S. McLaughlin, Biophys. J. 44 (1983) 325.

11. J. G. Kirkwood and J. B. Shumaker, Proc. Natl. Acad. Sci. USA 38(1952) 863.

12. L. Guldbrand, B. Jönsson, H. Wennerström and P. Linse, J. Chem. Phys. 80 (1984) 2221.

13. A. G. Moreira and R. R. Netz, Phys. Rev. Lett. 87078301 (2001).

14. J. Reščič, P. Linse, J. Phys. Chem. B 104, 7852 (2000).

15. B. Svensson and B. Jönsson, Chem. Phys. Lett. 108580 (1984).

16. B. Hribar and V. Vlachy, J. Phys. Chem. B 101, 3457 (1997).

17. P. Linse, J. Chem. Phys. 113, 4359 (2000).

18. K. Bohinc, A. Iglič and S. May, Europhys. Lett. 68(4) (2004) 494. 
19. S. May, A. Iglič, J. Reščič, S. Maset and K. Bohinc, J. Chem. Phys. B 112 (2008) 1685.

20. S. Maset and K. Bohinc, J. Phys. A: Math. Theor. 40 (2007) 11815.

21. J. Urbanija, K. Bohinc, A. Bellen, S. Maset, A. Igli, V. Kralj-Igli, P.B.S. Kumar, J. Chem. Phys. 129(10) (2008) 105101.

22. S. Maset, J. Reščič, S. May, J. Pavlič, K. Bohinc, J. Phys. A: Math. Theor. 42 (2009) 105401.

23. Y. W. Kim, J. Yi and P. A. Pincus, Phys. Rev. Lett. 101(20) (2008) 208305.

24. M. M. Hatlo and L. Lue, Soft Matter 5 (2009) 125.

25. M. M. Hatlo and L. Lue, EPL 89 (2010) 25002.

26. M. M. Hatlo, K. Bohinc and L. Lue, J. Chem. Phys. 132 (2010) 114102.

27. K. Bohinc and L. Lue, Chin. J. Polymer Sci. 29 (2011) 414.

28. K. Bohinc, J. M. A. Grime and L. Lue, Soft Matter 8 (2012) 5679.

29. K. Bohinc, J. Reščič, J. F. Dufreche, L. Lue, J. Phys. Chem. B 117 (36) (2013) 10846 . 